\title{
Indoor navigation systems based on data mining techniques in internet of things: a survey
}

\author{
Mahbubeh Sattarian $^{1} \cdot$ Javad Rezazadeh $^{1,2}$ (D) $\cdot$ Reza Farahbakhsh $^{3} \cdot$ Alireza Bagheri $^{4}$
}

(C) Springer Science+Business Media, LLC, part of Springer Nature 2018

\begin{abstract}
Internet of Things (IoT) is turning into an essential part of daily life, and numerous IoT-based scenarios will be seen in future of modern cities ranging from small indoor situations to huge outdoor environments. In this era, navigation continues to be a crucial element in both outdoor and indoor environments, and many solutions have been provided in both cases. On the other side, recent smart objects have produced a substantial amount of various data which demands sophisticated data mining solutions to cope with them. This paper presents a detailed review of previous studies on using data mining techniques in indoor navigation systems for the loT scenarios. We aim to understand what type of navigation problems exist in different IoT scenarios with a focus on indoor environments and later on we investigate how data mining solutions can provide solutions on those challenges.
\end{abstract}

Keywords IoT $\cdot$ Indoor navigation system $\cdot$ Indoor positioning $\cdot$ Data mining $\cdot$ Machine learning

\section{Introduction}

Internet of Things (IoT) can be deemed as a fast growing technology the fundamental idea of which is to connect all the physical devices. since we are living in a world in which many are seeking to create machines which can think independently, data mining has widely proved to make an IoT system smarter, which is a convincing explanation for the achievements of IoT.

Javad Rezazadeh

rezazadeh@ieee.org

Mahbubeh Sattarian

M.Sattarian@outlook.com

Reza Farahbakhsh

reza.farahbakhsh@it-sudparis.eu

Alireza Bagheri

ar_bagheri@aut.ac.ir

1 Tehran North Branch, Islamic Azad University, Tehran, Iran

2 University of Technology Sydney, Ultimo, Australia

3 Institut Mines-Telecom, Telecom Sud-Paris, 91000 Evry, France

4 Amirkabir University of Technology, Tehran, Iran
The IoT devices are able to provide safe, reliable and efficient solutions especially in high-risk Environment,health and safety (EHS), Montbel Thibaud et. al. have reviewed existing published research on IoT-based applications in EHS industries with specific emphasis on healthcare, food supply chain, mining, energy, intelligent transportation, building and infrastructure management [1]. We could mention many other IoT application such as face recognition using neural learning techniques [2] which could be useful for blind people to navigate them easily with the help of a smart phone [3, 4]. In all IoT applications, different types of data must be collected and analyzed [5], depend on the type of data, different techniques must be applied and make prediction so DM techniques should to deal with the high convergence of the IoT data which are quickly produced. In [6] DM techniques for IoT are examined. In [7] also present an approach for fusing noisy fingerprints with distance bounds for indoor localization.

On the other side, positioning systems have had a major role in the application for IoT including supply chain management and logistics [8]. These solutions have already been executed with RFID chips and can be categorized among the first IoT applications produced thus far. The capacity to identify a certain position in smart exhibitions 
and buildings is one of the most critical functions in that area. The primary objective of any smart home might be to categorize human activities at home or in an indoor environment. Noury et. al. [9] have demonstrated the capability of the today's smart home to identify human activities.Navigation and positioning via smart phones are highly useful in daily life.

The technology of GPS satellite started to be widely accessible in the late 90's. Nowadays almost all people possess a gadget with positioning functions such as a tablet, smart phone, GPS tracking device or even a watch having built-in GPS. The GPS greatly modified outdoor positioning. Despite this, GPS-based navigation fails working wherever visual contact to several GPS satellites is not available. Nor is it possible to figure out the floor level which a device is placed on. The biggest issues of these systems include regions of non-line-of-sight (NLOS) conditions, presence of high multi path propagation and low signal strength available. Today, positioning technology runs indoor creating various possibilities for indoor use.

More complications take place in using GPS for indoor positioning than for outdoor one. The main reason is that a particular infrastructure should be set up indoor. Research concerning indoor navigation systems (INSs) has increased due to the development of IoT, most of which relies on good wireless sources and signals. In complex facilities which consist of many floors like exhibition halls, airports, hospitals, railway stations, shopping malls, office and industry buildings, precise navigation such as road traffic navigation is needed. In cases of asset and staff tracking, location based services and Geo-fencing, an accurate location recognition is required. For positioning to work indoors, where individuals spend the majority of their time, new technological innovations are necessary. Smart phones having cameras can identify that pattern and look it up in an on line database to establish their position. Various positioning technologies are used by IoT to execute indoor navigation and indoor positioning. More and more studies are being conducted in this field.

Only fifty percent of the solution is determining the location of a person or device indoors. Service providers have to have accurate indoor maps, if the location is to be used for navigation or some other objectives. One of the main problems in dealing with complexity difficulties has always been formalization of the intuitive conceptual difference between the substantial number of variances in relationships existing in random collections, and sometimes the relationships between elements in systems in which constraints (associated with correlation of otherwise independent components) concurrently lessen the variations from element independence and produce identifiable regimes of more-uniform relationships, or interactions. These results have ambiguities and irregularities which make it complicated to understand. Traditional database technologies were not intended for huge data volumes, in addition database technologies are not fast enough to assess data.

Data mining can be explained as a process in which examining data takes place with the purpose of finding out patterns and trends. It checks databases for covered patterns, thereby discovering predictive information that specialists might overlook simply because it is placed beyond their expectations, DM creates a perspective to showing a path that will enable us to offer advantages in an effective way and concentrate on the issues, a significant piece of this value equation is the time and cost it usually takes to produce new statistics to the issue. Data mining can be applied in numerous areas of Indoor Positioning system in order to estimate and find out trends.

It is a practical solution for looking to obtain a competitive edge. In the past, we could only assess what INSs Devices or clients had done. Today, however, thanks to data mining, we are able to forecast what client will do. With data mining, INS can make more successful decisions in advertising and marketing. Such decisions will also allow INS to expand. Robust and Accurate positioning in indoor environments is a demanding job, particularly because of the modeling of the environment that can carry out large-scale models of the environment and the tag population and the usage of VAs for localization permits to identify the position of a certain target with using only through one physical anchor node.

This technique of localization has been called multipath-assisted indoor navigation and tracking (MINT). Besides the MINT approach, synergy amongst users was presented in [10] to get over the problem of a multi modal possibility because of data-association ambiguities. Distance measurements between cooperating users have been traditionally used to non-cooperative MINT measurements to a known anchor node. No specific study on surveying data mining techniques in Indoor Navigation System exists. This study considers accurate and robust Indoor Localization as a major enabler of potential Location-Base Services.

Real-time interaction between users, objects, and Indoor Navigation systems, which includes the need for accurate recognition of activities and detection of objects, is the main function this kind of applications demander. This survey paper concentrates on DM techniques for Indoor Navigation Systems. As opposed to earlier studies, the primary focus of our study is on publications which meet a number of criteria. This paper is expected to be useful for those readers who intend to start research in the domain of DM for Indoor Navigation System. As a result, much emphasis is put on a comprehensive explanation of the DM 
techniques and references to how these techniques have been applied in Indoor Navigation Systems.

The rest of this report is arranged in this way: Sect. 2 centers on major Indoor Navigation System Problem and solution. Section 3 discusses data mining techniques. Data mining includes a route to knowledge discovery [11] and is a significant process to uncover patterns in data by investigating and modeling substantial amounts of data [12] The difference between statistics and data mining has been ascribed to the features of the analysis; statistics is related to primary analysis, while data mining handles secondary analysis that is obtained from data. Data mining uses machine-learning algorithms to learn and recognize valuable information and following knowledge from substantial databases [13]. Section 4 explains challenges and future work, and finally, Sect. 5 concludes this study.

\section{Indoor navigation systems}

Today in mega cities, the job of transferring people to different places and orienting them in complex spaces is very challenging. Since electronically technological tools have been highly advanced, Japanese authorities have tried to use navigation systems such as GPS and cell phone for visually impaired people [14]. On the other hand, many initiatives were presented making use of various technologies such as GPS, GMS, Ultrasonic, RFID, and Laser [15]. A detailed analysis of different indoor positioning methods together with their operational technology can be seen in [16]. There, agents discover their positions using temporal and spatial synergy to obtain information from various sources. The existing framework provides a great insight into the evolution of joint information in networks of navigation based on the Fisher information matrix (FIM). [17]. A famous framework for theoretical analysis and interpretation of information fusion in networks are displayed in [18]. We, thus, focus on the recent developments which are closely relevant to the present research.

It worth to mention that navigation and localization can be coupled or decoupled, based on the specific application scenarios. The former may not necessarily rely on the latter technique. A nice summary of navigation and localization is presented in [19] and worth to mention, Travi-Navi that enables a self-motivated user with out comprehensive indoor localization systems was implemented in [20].

The technologies used in INS are summarized in Table 1. the easiest localization method which is accessible in cellular networks is The Cell ID (which is Cell-of-origin). Many other methods exist which make use of angles of radio signals and/or time (distance). Some of them are more known than others including: TDoA (Time Difference of Arrival), AoA (Angle of Arrival), and ToA (Time
Table 1 Indoor navigation technologies

\begin{tabular}{lllc}
\hline Technology & Based on & Position method & Accuracy \\
\hline Cell-ID & Network & TDoA, EOTD, RSSI & $200-5000 \mathrm{~m}$ \\
Cell tower & Network & Triangulation & $50-1000 \mathrm{~m}$ \\
IMES & Based radio & Navigation massage & $3-10 \mathrm{~m}$ \\
WIFI & Infrastructure & ToA, signal strength & $5-15 \mathrm{~m}$ \\
UWB & Infrastructure & ToA & $10-30 \mathrm{~cm}$ \\
Bluetooth & Infrastructure & RSSI fingerprinting & $3-10 \mathrm{~m}$ \\
\hline
\end{tabular}

of Arrival). IMES can effeciently produce 3D positioning data using a transmitter unit without calculating range. In addition, even in deep indoor locations, IMES is highly accurate. In contrast, GPS, A-GPS, and cell tower fail to produce data with similar quality in this kind of areas. One of the short-range radio technologies applied for indoor positioning Ultra-wide band. As opposed to Wi-F and Bluetooth Low Energy, positioning can be performed with transit time methodology (called Time of Flight) rather than calculating signal strengths (Receive Signal Strength Indicator).

In this method, the running time of light between an object and many receivers will be calculated. Similarly, Blue tooth and Wi-Fi technologies have used the same methods known as triangulation and cell-id, but they use them in wireless hot-spots. Two factors determine the accuracy finally obtained: the location-positioning algorithms to be used and density of access point. In addition, various systems have been created to position objects which are inside the building. Each of these systems has their own advantages and disadvantages. For instance, infrared is the main pattern in receivers and transmitters of such systems. There are two main disadvantages. The first one is that infrared makes use of direct-view and shortrange navigation.

As a result, signals transmitted by 802.11 networks can readily cause inaccurate communication. recently, in order to locate some objects in covered areas, they have tried to combine GPS and wireless network including $\mathrm{Wi}-\mathrm{Fi}$, mobile communication, RFID or UWB. The use of Wi- Fi was almost effective, but not enough, due to the fact it needs GPS signals to tag Wi-Fi data collected through mobile phones. yet, if you are encircled by an environment which is full of Wi-Fi signals, those are useless unless they are combined with GPS signals. To avoid this issue, a research team was created to develop a radio map that was based only on the effect of the Wi Fi signals. They were successful in devising a new method to build Wi Fi radio maps that do not need GPS signals. WIFI effect pattern, set of WIFI signals received by a cell and RSSs around the 
device. The WIFI radio link show RSSs network access points at various point in a geographical area. This system is called INS. it lowers the loss and improves simplicity of the operation.

The best description of INS is that it is much like GPS for covered environments. This system can determine the location of objects or people in a building making use of radio waves, magnetic fields, audio signals or other sensory information obtained from mobile devices. Using the WIFI detector can help to determine the positioning of signal transducers in a closed environment with a accuracy of about 4 to 6 meters. The basis of performance of this signal is based on the imitation of the detection of radio signals of the environment. The internal positioning system, which make use of the transmitter and receiver of WIFI waves in 35 channels from $2.54 \mathrm{GHz}$ to $5.8 \mathrm{GHz}$, can compute the distance between receiver and transmitter with the difference in waves motion in the environment. WIFI range is about 20 meters. Today, regarding the availability of WIFI signals, they use it as the resource to identify the location of buildings.

INS is necessary in places like hospitals, shopping malls, and other indoor halls where location-based services can be helpful. The INS technology through the internal sensor lever on smart phones, computes the internal position of the device utilizing complex mathematical algorithms. A combination of received data from this sensor and clever ways can be calculated in a very accurate position.

One of the common positioning systems is the GPS. However, GPS positioning is not suitable because of the nature of technology used for indoor applications, because to communicate with satellites, it needs clear and unobstructed field of view [21]. In other words, walls and other objects can weaken its signal availability (Table 2).

In current years, using GPS and wireless networks such as WIFI, mobile communication, ultra-wideband (UWB) or radio frequency (RFID) have been considered to locate uncovered areas. The use of WPS technology is to some extent beneficial but not enough because it needs GPS signals to tag the WIFI information collected through mobile phones. Still, if you are surrounded by an environment which is full of WIFI signals, these signals are useless unless they are combined with GPS signals.
To overcome this issue, they in fact could devise an innovative way to build a WIFI radio map that does not need GPS signals. The WIFI effect pattern is a set of WIFI signals received by a mobile device and the measurement of the power of the received WIFI signals (RSSs) from the available points around the device. The radio map of WIFI, RSSs displays network access point in different geographical regions. This system is referred to as IPS, Which minimizes waste and makes operation simple. The best description for IPS or INS is to point out that it is a technology very much like GPS but for indoor environments. This system employs magnetic fields, audio signals, radio waves, or other types of sensory information obtained from mobile devices to locate objects or identify people who are in a building. By using WIFI environment detector technology, it is possible to find out the positioning of signal transducers in closed environments with 4-6 meters accuracy. The basis of this signals performance is based on the imitation of the detection of radio signals in the environment. The internal positioning system, using 35 channel WIFI transceivers and receivers, from $2.4 \mathrm{GHz}$ to $5.8 \mathrm{GHz}$, that able to calculate the distance between the waves. The Wi-Fi connection is about 20 meters. Recently, as for the availability of Wi-Fi signals, they use it as access sources to identify the location of the buildings.

Indoor navigation as an autonomous system that normally has five principal components [34] including mobility, localization, navigation, planning, and communication, We briefly explain each of these components below.

\subsection{Mobility}

The mobility can be viewed as the impact of the global system. In fact, it is the outcome of system observation in its entirety [35]. in order to support indoor navigation and localization, an integration between directional measurements and the physical displacement at low level is required and increased with location context into more complex human mobility information, for example locomotion modalities, trajectories, walking steps, virtual landmarks including lifts and stairs, etc [22]. Mobility can enhance the accuracy of localization, reduce the cost of deployment, and improve location context [8]. The primary
Table 2 Major components of indoor navigation systems

\begin{tabular}{lll}
\hline Component & Description & REF \\
\hline Mobility & Physical displacement and directional measurements & {$[22-27]$} \\
Localization & Exclusive function of exit in relation to the rest of system & {$[28,29]$} \\
Navigation & The process of monitoring and controlling the movement & {$[30]$} \\
Planning & Produce adequate navigation commands & {$[31,32]$} \\
Communication & Provides the link between all the possible elements & {$[33]$} \\
\hline
\end{tabular}


goal of the system is to present valuable information about navigation which will allow a user to make proper and well-timed decisions based on which he can follow a path in an indoor space. A number of intriguing proposals have been offered in mobility and location support field. The focus of the projects is placed on: outdoor [23], indoor [24]. The studies which have dealt with indoor environments have offered many ad hoc technologies and strategies used in order to offer valuable information to user [14]. For instance, Sonnenblick [24]. executed a system of navigation used for indoor environments according to infrared LEDs mechanism. Radio Frequency Identification (RFID) is a technology commonly used. A considerable number of studies have been carried out to identify users position and carry out tracking of object in indoor environments with support of fingerprinting with blue-tooth low energy beacons [26, 27].

\subsection{Localization}

The process of determining physical coordinates of a sensor or the spatial relationships among objects is known as localization [36]. Information about location offers a great deal of data which includes speed, attitude, and acceleration of the vehicle. The location can be viewed as an exclusive function of exit with regards to the other parts of the system. This indicates that the boosting the capacity by location can usually move forward separately from the other system elements. Localization divided into target source localization and node self-localization. The majority of the methods of source localization emphasize the strength of signal measured. Additionally, node calculation is a highly complex process [8]. The focus of the present study is on patterns matching localization which is also referred to as map base or finger print algorithm. It includes two stages: (1) at the chosen locations, received signals are recorded in radio map which is an offline database. (2) It works in online way. The pattern matching algorithms are applied to identify the unknown node location by corresponding the existing observed signal attributes to the prerecorded values which are on the map [28]. Localization may be categorized regarding different criteria: (i) Centralized or Distributed (ii) Range-free or Range-based and Learning-Based or Filter-Based Techniques (iii) Mobile or Stationary, (iv) Coarse-grained or fine-grained. Location of sensor readings to identify the event is accuracy, coverage, and time [37]. GPS Constellation of 27 satellites (24 active and 3 redundant), each node with GPS is expensive for sensor node because of infrastructure, problem of form factor, requires line of sight and synchronized clock. According to [29], the techniques of localization may be broken down to two broad groups, namely centralized and decentralized, based on the structure of nodal organization.
Decentralized localization can be also divided into two groups depending on how we calculate the locations existing between each node. These consist of range-based techniques as well as range-free techniques, as displayed in Fig. 1.

Quite a number of localization techniques which are employed in the field are among range-based techniques. They can be categorized with regards to their features, including distance and angle [38, 39]. In addition, rangefree techniques may be categorized through counting the local hop [40]. Likewise, localization methods are categorized into two groups namely anchor/beacon-based or anchor/beacon-free; GPS-based or GPS-free; coarsegrained or fine-grained; and mobile or stationary sensor nodes, among many others [41]. It is evident that the Beacon placement can highly impact the accuracy of localization [42]. Amongst all the methods, Wi-Fi fingerprinting has come forth as an appealing method which is because of the deploy capability of it. Normally two stages exist in fingerprint-based localization, including off line site survey and on line location query. In the off line stage, a site survey is carried out in order to gather the fingerprints at recognized physical locations referred to as reference points (RPs). Each fingerprint is in fact a vector of received signal strength (RSS) values of Wi-Fi access points (APs). The RSS values and their related locations are subsequently filed in a database. In the on line stage, however, the RSS values are measured by a mobile client (target) at its location. After obtaining the measurement from the client, the server starts matching it with the most identical fingerprints and sends back the client location. How close the fingerprint database matches with the existing signal environment will determine the accuracy of fingerprinting localization. Any time AP signals are modified via AP movement or power adjustment, can no longer achieve adequate accuracy will no longer be obtained by conventional fingerprint based indoor localization schemes [27].

\subsection{Navigation}

Navigation constitutes a field of research which mainly deals with the procedure of controlling and monitoring the movement in vehicles and crafts from one location to

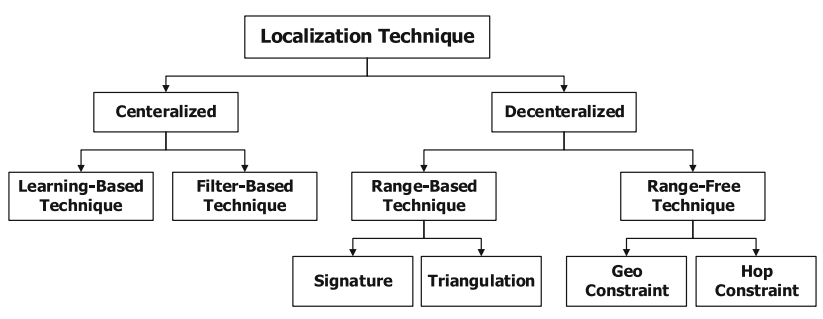

Fig. 1 Sub-categories of localization techniques 
another one [8]. Additionally, the navigation is related to the obtaining data and responds to information from the external environment. Put simply, the receivers can collect the data via the observation done by the sensors in the operational environments. This observation is very critical, due to the fact it results in an internal interpretation of the environment, which can be utilized later during the projects [36]. One of the good examples of LBS navigation is the mobile phone applications such as Google Maps. Real-time tracking can be considered as one of the most beneficial applications of LBS in this regard. The LBS approach employed to navigate provides the distinct benefit compared to mobile navigation software currently using data stored on the memory of a mobile device, since it sometimes provides access to the real-time data. The limited amount of data transferred via wireless networks has been underestimated, as not only do several network operators provide unlimited range of data, but also it is more costeffective [30].

\subsection{Planning}

Planning has a critical role to create the trajectories, behavior and/or points of passage for the system [8]. Planning has no direct connection with any controllers of entry or exit sensory. Despite this, having substantial knowledge of map reading and can resolve the upcoming situations to produce sufficient navigation commands. In short, introduced indoor positioning systems use whether sensing technology or the information obtained from multiple information sources. These can provide a better solution to multi-path sources and merge information from multiple information sources respectively [31].

WiFi fingerprinting can be regarded as the most common method for indoor tracking and localization. Several modern systems are dependent on fingerprinting algorithms. These kinds of algorithms operate in two distinct stages, namely off line or on line. First of all, the off line stage, is utilized to acquire measurements which are obtained by signal strength (the fingerprints) from all recognized basic stations at predetermined locations. The values are put into a radio environment map. As the second stage, which is the on line stage, the information related to signal strength is constantly assessed and compared to a database containing all fingerprints taken from the off line stage [32].

The software of analysis might be put into present indoor positioning/indoor navigation systems client-based or server-based or assembled independently. The end user devices are identified by the technology. Ultra-wide band, Blue tooth transmitters, and $\mathrm{Wi}-\mathrm{Fi}$, calculate and label the time used in each location and find out which routes were utilized. As an example, this allows you to understand the number of people who have crossed a specific area during the day or which hours have been the least busy. The object movements, e.g. work equipment, or products may also be assessed as well as the visitors' walking paths. The data is shown in the form of diagrams and radio environment maps.

\subsection{Communication}

The communication offers the link between the vehicles and all possible components of the global system, such as other vehicles and/or operators. At the moment smart phones, navigators and other devices held in hand offer location-based information in order to support navigation while moving outside buildings. Inside buildings, however, finding the way and navigation patterns become even more difficult than ever due to the fact they cannot be tracked by GPS. Many different technological instruments have been suggested for indoor positioning. Current indoor positioning systems are making use of the technology of radio frequency (RF) like WLAN, ultra wide band (UWB), radio frequency identification (RFID), Blue tooth, and ultra-high frequency (UHF) [33] Indoor Positioning technology can be categorized by the physical principles of their sensors as well: (i) optical methods, e.g. time-of-flight (ToF) cameras, (ii) acoustic methods, e.g. ultra sonic measurements using ToA, (iii) inertial measurements, e.g. acceleration measurements comprised in an inertial measurement unit (IMU) for dead-reckoning and (iv) radio-based measurements, e.g. GPS using TDoA.

\subsection{Commercialized solutions in INS}

Recent Advancement in the intelligent building industry has made convenience and well-being with energy-saving. As a potential result, people spend more time in internal environments, the precise positioning of a user within a building is increasingly important for public safely and other services. This has made INS techniques a useful application for many applications, and large technology companies are trying to make more use of it in their services and make it even more effective. for instance, Google has used INS in their Tango project [43] to enhance user experience in the local environment, including many Indoor Localization and Navigation techniques. Augmented Reality (AR) framework that digitally supports facility maintenance operators when navigating indoors, is another powerful method that is highly connected to the real world which works with the Google map to access accurate spatial information inside the device. This technology uses mobile phones with the potential to provide indoor position at a centimeter level and combines computer vision, inertial tracking and machine learning [44]. 
There is a set of services that use existing sources of signal such as Wifi or Blue tooth to design the interior. Various companies like Google, Microsoft and Apple have developed similar technologies in a number of hospitals, shopping mall, airports and convention centers which a summary is presented in Table 3.

\subsection{Open issues and current solutions}

This part comprises some of the major open issues in the field of indoor navigation. A number of solutions exist which could help navigation and orientation become closely linked with the technological developments.

Today's smart phones, navigators and other hand held devices provide location-based applicability to support navigation when moving outside buildings. The comprehension of existing knowledge and understanding of hidden patterns among data have become far more from reality over the last few decades. Despite this, you can enter the IoT world and analyze the received information by installing modules which could apply various relevant IoT technology-based tools, such as radio frequencies, Wi-Fi, Blue tooth and so on. After analyzing the data, broadcasting the relevant commands is the next stage, either through machine learning or predetermined commands. Finally, it is expected operators follow the same rules.

As a subset of IoT, we can refer to INS where locationbased services are necessary. The accuracy of this method is determined by the amount of information obtained. We can try to examine and enhance the performance of M2M via data mining, which is one of the most powerful and well-known techniques of data analysis. We also seek to find hidden pattern among data by using statistical models, and, finally, finding valuable connections between data elements is the last activity should be taken into consideration. Data mining prioritizes and weighs important indicators. It initially prioritizes the quality indicators to provide a more accurate and suitable configuration for each situation. In this case, appropriate decisions can be made for each situation, which will speed up its feasibility and avoid any errors. What is more, it has some benefits such as resource management and coordination. So based on data mining techniques, examining the attributes and features of the patterns gathered in a central server which could be obtained from the covered areas like hospitals, shopping malls or airports by using a history of these behavioral patterns. There is an enhancement on the INS patterns, and, therefore, we can reduce the location errors and provide a better way than before.

(1) Accuracy The major limitation of mobility is when a certain object in the environments mentioned is moving, the map has to be refreshed in order to properly modify the algorithm employed for detecting the objects and the movement of the users in the environment. This process will be based on the existing situation. Still, various types of algorithms might be examined to enhance the system accuracy. [45, 46].

(2) Complexity Another important matter is, in the public areas for example commercial centers, airports, or university campus, in which using an accurate Radio Map from mapping system is essential. Additionally, issues with scalability may arise from the trade-off between the diversity of samples and time-to-response, Lastly, when this positioning systems is used in common smart phones to guide people, some items still remain inconsiderable including magnetometer hardware, users velocity, and device orientation [47, 48].

\section{An overview on data mining techniques}

Technologies and methods are produced by data science which can be employed in order to develop data analytics and machine learning instruments intended for managing tasks and analyzing network. Furthermore, they can provide a springboard from operator data assets into lucrative use occasions. We can create dynamic models only when big data sets with concurrent configuration background as well as a maintenance log over long periods of time are available. They help to record the main issues and update whatever happening in the network. Without having the history of all the events, a domain expert or data scientist will be unable to know whenever the findings make any sense or not.

Table 3 Commercial indoor navigation company

\begin{tabular}{llll}
\hline Characteristic & Google & Microsoft & Apple \\
\hline Main solution & Use deep detection technology & Focus on orientation rather than positioning & iBeacon protocol \\
Advantage & Low energy consumption & Low cost & Compatibility \\
Product & Tango/AR & Path guide & AI/AR \\
Base on & WiFi trilateration & Geomagnetism and natural walking patterns & WiFi and smart phone sensors \\
\hline
\end{tabular}


By a broad definition of the functionality of data mining, we can say it includes the process of finding useful knowledge from huge amounts of data kept in either data warehouses, databases, or other information stores. DM is actually a specific stage in the mentioned process which includes the use of certain algorithms for taking out patterns from data. The additional simplify process (decrease noise in data, decrease dimensionality, find critical factors or combinations) assure that valuable knowledge is taken from data. Yet, there exist a number of publications (e.g., Cross Industry Standard Process for data mining (CRISPDM)) [13] and industry participants who consider the whole process of KDD as DM. In Fayyed et als study [49], DM is employed to explain a certain step KDD that handles application of particular algorithms for taking out patterns from data. DM focuses on the discovery of formerly unidentified attributes in the data and does not require a particular goal from the area and finding new knowledge. Some famous data mining platforms are Weka [50], R [51] and Rapid Minder [52] support data mining models.

A considerable overlap exists between ML and DM. Many might confuse these two terms for the reason that they usually make use of the same methods and consequently overlap substantially. ML as an area of research that offers computers the capability to learn with no specific programming. ML concentrates on categorization and prediction, based on known qualities formerly discovered from the training data. ML algorithms require a goal (problem formulation) from the domain (e.g., dependent variable to predict). DM concentrates on the discovery of formerly unfamiliar attributes in the data. It does not require a specific goal from the domain, but rather concentrates on discovering fresh and helpful knowledge. The model that ML executes should be based on factors in dataset, and not to be fine-tuned based on its accuracy in the test data set. Two general approaches exist in this regard: The classical statistics which is simple to understand. The other approach is modern machine learning which is more complicated and requires much computing power. In data mining both of these funded as shown in Table 4.

Table 4 Data mining algorithm

\begin{tabular}{ll}
\hline Classical statistics & Machine learning \\
\hline Linear regression & Hidden Markov models \\
K-means classification & Support vector regression \\
K-Nearest neighbor & Random forests \\
Hierarchical clustering & LASSO regression \\
\hline
\end{tabular}

Those operators have achieved more success at benefiting from the power of big data who have data analytics as integral part of their approaches. such skills enables us to create a decision-making culture which is based on data across the whole organization. The benefit of saving volumes data will depend on our capability to draw out helpful information, identify exciting incidents, assist policies and decisions based on statistical evaluation, and manipulate the data to attain operational, scientific, or operational objectives. Data mining includes four operations: Clustering, Classification, Association analysis, Anomaly detection. The purpose of Clustering is find groups which are comparable to each other and the point here is that classification here is pragmatic. Classification is the process to select the proper path and consists of processing again, assessment and affirmation of features, and model design. Classification is a method which harmonizes with clustering and is regarded as an example of Supervised and unsupervised Machine learning. If we understand what items have to be presented to the computer in order to identify Supervised or labeled learning are be employed, where in the data have a true class or outcome variable accuracy is the guiding concern. Therefore, we can affix labels to these samples of data and deploy the machine to understand features. Unsupervised methods are effective when data dont have any proper groups or criteria so groups are shaped based on similarity not accuracy. They can show correlations, trends, clusters and other types of information. Research about algorithms for classification is an interesting area due to the fact there is a vast range of options that are very interoperability for instant we summarized them in Table 5.

\subsection{Knowledge discovery}

In Internet of Thing research field, distributed computing is utilized to split bulk data issues into smaller sections and parallel processing of the main node to some of more compact subproblems, and a working node delivers several smaller problems. In fact, it processes the workers node and keeps the outcomes in local file system. After that, the input data is assessed and combined from the mapping phase. Knowledge discovery from the data consists of different analytical techniques including data mining, modeling, statistical analysis, interactions and visualization. Statistical analyses deals with outlining large bulk datasets, understanding data and determining models for prediction. Data mining is concerned with discovering important patterns in the massive data sets which are related to them, machine learning combined with data mining, and statistical methods that allow machines to recognize data sets. Visual analysis method, the area in which the massive data set will serve users, in ways that are 
Table 5 Classification algorithms

\begin{tabular}{ll}
\hline Algorithms & Description \\
\hline Decision tree & Find variables and values that best split cases at multiple levels \\
K-NN (K-nearest neighbors) & $\begin{array}{l}\text { Designed to find the k cases in multidimensional space closest to the new one } \\
\text { Naive bayes }\end{array}$ \\
Adjust probability with each new piece of information \\
Supdom forests & A collection of decision trees and randomly select cases and variables or features \\
Artificial neural networks(ANN) & Uses the "kernel tick" to find a hyperplane that cleanly separates two groups \\
K-means & Multiple layers of equations and weights to model nonlinear outcomes \\
Logistic regression & Associated learning algorithms that analyze data and recognized patterns \\
\hline
\end{tabular}

difficult, will be able to understand the relationships. Discovery of bulk data includes some operators developed to receive information from complex data sets. The general model of knowledge discovery from bulk data has nine phases:

1. Apply first to application information and define the purpose of processing from the users perspective.

2. Create a subset of data that refers to the discovery of knowledge.

3. Removing noise, managing lost data fields, acquiring information required for modeling and calculating information, time and major changes.

4. Finding important attributes to demonstrate the dependence on the purpose of the work.

5. Mapping targets in accurate data mining methods.

6. Select the algorithm related to data mining and function to look for data patterns.

7. Searching for patterns in a rational way.

8. Return to each of the steps 1 to 7 for repetition and recitation, as well as this stage may consist of illustration of the patterns.

9. Using information in a direct way, merging information into another system or reporting and using information in a simplified way.

\subsection{Network analysis process (ANP)}

A more typical form of the analytic hierarchy process (AHP) utilized in multi-criteria decision analysis is called the analytic network process (ANP). AHP structures decisions and problem into a hierarchy target, alternatives and decision criteria, while the ANP structures it in the form of a network. Thus, both of these process employ a system of two-by-two evaluations in order to determine the weights of components structure, and ultimately to evaluate the alternative decision. Thus, ANP has all Asps positive attributes, which include simplicity, flexibility, application of quantitative and qualitative criteria simultaneously, and the ability to review compatibility in judgments, and in addition, it can take complex communications (interdependencies and feedback) between and among decision elements by using a network structure rather than a hierarchical one. [53] All components in a network can be connected together in any form. In other words, it is possible in a network of feedback and interconnection among clusters. As a result, the ANP can be broken down into a number of parts: Control hierarchy, network communication, relationship among criteria, goal, and sub-criteria which can affect the systems intrinsic interactions and consists of a network connection between elements and clusters. The complex relationship between decision elements is considered through a hierarchical substitution with a network structure. The network analysis process comprises the four stages:

\section{Design the model and transform problem into network structure}

The subject should be moved to a grid in an open and clear way. At this point, the problem is converted into a network structure in which the nodes are presented as clusters. Elements within a cluster can have one or all of the elements. Other clusters are interconnected. It may also be possible for elements within a cluster to interact with each other themselves.

\section{Creating a binary matrix and identifying priority vectors}

Just like binary comparisons in AHP, the decisions and elements within the clusters are compared based on their significance with regards to the criteria of two-way control. The clusters themselves are also based on their roles and effects on accomplishing the goal are compared. Furthermore, the interdependencies between elements of a cluster should also be compared. The effects of each element on another elements can be represented through a special 
vector. In this section, the internal importance vectors computed to indicate the relative importances (coefficient of importance) elements with clusters, which is obtained through the following equation: $A W=\max W$, where in: $\mathrm{A}$ : binary comparison matrix criteria, W: special vector (coefficient of importance), max: the greatest of all) is numeric [54].

\section{Generate super matrix and convert it to super matrix Limit}

To accomplish the general priorities in a system with interactions, the main privilege vectors (W calculated) are inserted into the equivalent columns of a specific matrix. Therefore, each section of this super matrix (a partitioned matrix) exhibits two clusters in a system are related. On the next step, the superconducting matrix is obtained by multiplying the unbalanced cluster super matrix it will be counted. Then, by normalizing the super matrix, the column is converted to a random state. As the third and last step, the super matrix is bounded by the ability to bring all the super-matrix elements to the point where divergence is achieved (through repetition): $\lim _{K \rightarrow \infty} W^{K}$.

\section{Choose the best option}

In case the super matrix is composed in the third step, the whole network is taken into consideration, that is, options are also included in the matrix, general priority of the options is acceptable from the columns option in the normalized super matrix. If the super matrix consists of only part of the network that is interdependent, and the options are not considered in the super matrix; further calculations are required for obtaining the priority of options. The option with most general priorities are considered as the best options selected for the topic.

\subsection{Deep learning impact on indoor navigation}

Deep Learning (DL) techniques inspired by the structure and function of the brain called artificial neural networks. DL applications are transforming several existing problems, and INS might not be an exception. Various DL projects are launched in different domains from medical services to marketing and image/video processing. One of the most important area of DL application is working with the text which Googles DL RNN keeps improving on it, another important field of DL application is related to speech and image processing. In [55] and [56] overcome to these limitations has been shown. This approach provides a fast and efficient method for tracking in the environment, which is often more useful for applications than static localization. it is also a live deployment suitable combination approach, where some algorithms capable of delivering a precision of 0.76 meters. Over time, many systems require an intelligence and ability to derive new knowledge from past experiences. Indoor positioning based on fingerprinting has attracted significant interest due to its high accuracy, in [57] a novel deep-learning-based indoor fingerprinting system using channel state information is presented which their experimental results are a confirmation of the Deep Learning ability to reduce location error effectively. There are other ongoing researches in this field for instance, a conventional Neural Network (CNN) with Deep Learning is approached in [58] to identify and localize vehicles in a car-park. Also the use of deep learning for image classification, object detection, segmentation, registration, and other tasks has been presented in [59].

\section{Data mining techniques in INS}

\subsection{Data mining techniques for navigations}

Data management can be considered as an extensive process which include many other data disciplines. Therefore, big data management is a complicated procedure. Thats why big data management has turned into an essential element for accessibility for decision making and thus enhance productivity. Additionally, storage management is very crucial in data management the context. These kinds of methods were created and implemented in order to execute clustering, replicating and indexing of data. Studying big data directly suggests greatly time-consuming navigation in a huge search space to present suggestions and feedback to customers. Environment has specific use for examining complicated earth system simulations with big numbers of datasets. Data Management related to data storage, pre-processing, processing for data management process flow as can be seen in Fig. 2 based on scalability, integrity, and availability. Managing big data calls for more

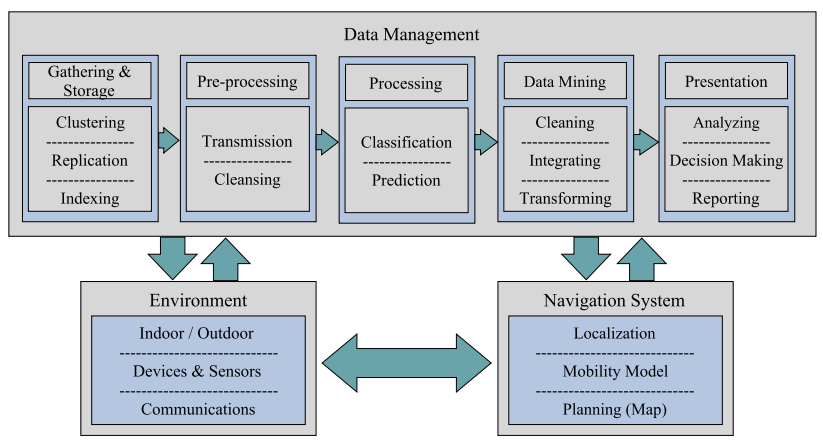

Fig. 2 Data management essential elements for decision making 
effective techniques and instruments to support trend of big data. [60]

INS normally relies on three distinct elements as shown in Fig. 3: (i) the platform of infrastructure positioning system, (ii) the sensors which broadcast signals received by smart phones after the boost to the positioning system, (iii)and applications which are at the top of positioning system and by adding critical information, add value to the system.

As it can be seen in the Fig. 3 an implemented structure in a covered area in which information is exchanged via IoT devices and finally collected in the central server. Sensors of IoT transmits specific data objects to nearby smart devices in the area with help of radio signals. Identifiers data can be used to recognize the physical location of the device, track users and etc. The devices send signal to users mobile application and show the corresponding predefined content. In this structure, as it is clear, adequate data collection is possible; this information is collected in the central server and in the end data mining extracts useful information from raw data; these data remain unknown before the data mining operation.

One of the most critical jobs in data mining is to choose the proper data mining method. Data Mining methods must be selected depending on the kind of problems. A general approach must be employed to enhance the accuracy as well cost effectiveness of using data mining methods. Generally there are three main data mining methods which are reviewed here. Many other data mining methods exist, but the three ones reported here are regarded as more commonly applied by INS Problem. Data Mining Technologies for INS Mainly do their calculations on the server so approaches to collecting and providing information is from the perspective of database. Then for producing data use the User device abilities. The KDD process in INS is to draw out knowledge from data in the database context. The knowledge discovery process normally involves three steps:

(i) Data preparation: make the data ready for the purpose mining. It consists of 3 sub stages: combine data in different sources of data and

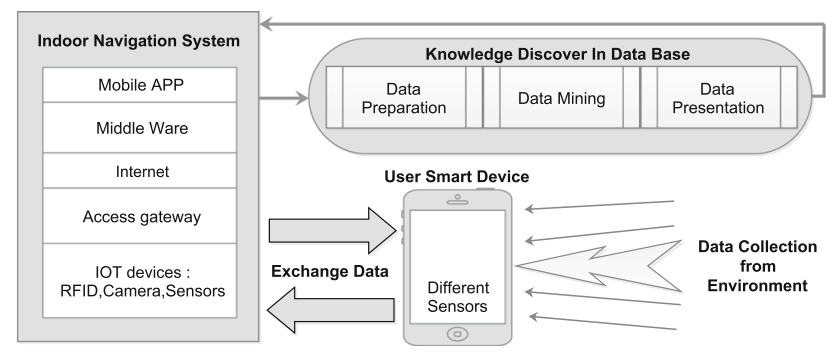

Fig. 3 Data mining processing for indoor navigation system remove the noise from data; Decide what data may be utilized with regard to the knowledge discovery; process the data to help data mining. It can include sophisticated statistical techniques, or making use of a data mining algorithm in this situation, selecting the data mining algorithms involves choosing method(s) to be applied for looking for patterns found in the data.

(ii) Data mining: use algorithms to the data in order to discover the patterns and examine patterns associated with discovered knowledge. This stage is vital for the good results of the whole KDD project. However, it is often highly projectspecific.

(iii) Data presentation: perform visualization on the data and present mined knowledge to the user. Data mining consist of clustering, classification, time series analysis, association analysis, and out lier analysis. In this stage we may have to utilize the algorithm many times till satisfactory result is obtained.

\subsubsection{INS problems}

As displayed in Fig. 3, each of the WiFi effect patterns on the radio map is linked to the devices location information or locations. Employing modules in mobile platforms and applications, we can gather the patterns effects and assess their functions and features. The accuracy of locating this method is determined based on the total of information gathered and when the amounts of informations gathered reaches more than $50 \%$, the average error of locating will be less than 10 meters. We presented some of the INS Problems and solution in different data mining methods on the Table 6. As it shows some previous solutions for accuracy mentioned including offers a route forward that is present for several, but not all, terminals [61], deal with the iBeacon placement strategy regarded as critical issues such as step detection placement strategy' considered important issues such as step detection [62]. In a number of IoT application, it is essential to understand the accurate or approximate location of the network component. Obtaining locations information is vital in many applications, and it pertains to Location Base System, in commercial applications, LBS describes an added value that provides information about the location of an object with the support of GIS [63].A control theoretic approach namely particle filtering was proposed [64], new visual vocabulary merging with expanded image processing methods [65], enhanced algorithm of fingerprint matching, which decreases interference by Gaussian filter [66], The pseudo lite system due to its great flexibility, strong signal power and high 
Table 6 Main metrics on indoor navigation systems, previous solutions and DM benefits

\begin{tabular}{|c|c|c|c|}
\hline $\begin{array}{l}\text { INS } \\
\text { challenges }\end{array}$ & Metric description & Previous solutions & Data mining solutions \\
\hline Accuracy & $\begin{array}{l}\text { Average euclidean distance between the } \\
\text { estimated position and the true positions }\end{array}$ & $\begin{array}{l}\text { Focuses on node placement [61], beacon } \\
\text { placements [62], Proximity based } \\
\text { localization [64], Fingerprinting } \\
\text { emerges as a straightforward and } \\
\text { plausible [69], novel indoor positioning } \\
\text { method [65], fingerprint matching } \\
\text { algorithm [66], Pseudo-lites [67] }\end{array}$ & $\begin{array}{l}\text { Generating an accurate Radio Map from } \\
\text { mapping, The data mining goal states } \\
\text { project objectives in business terms } \\
\text { could be defined as achieving a certain } \\
\text { level of predictive accuracy [70, 71], } \\
\text { Boosted Regression [72, 73], The } \\
\text { transparent data-mining model called as } \\
\text { DT and a black box model known as RF } \\
\text { [74] }\end{array}$ \\
\hline Availability & $\begin{array}{l}\text { Low availability (if }<95 \% \text { ), regular } \\
\text { availability (if between } 95 \% \& 99 \% \text { ), and } \\
\text { high availability (if }>99 \% \text { ), availability } \\
\text { might be affected by some factors such } \\
\text { as communications congestion and } \\
\text { routine maintenance }\end{array}$ & $\begin{array}{l}\text { Nonparametric Gaussian Process (GP) } \\
\text { model is adopted to describe the } \\
\text { relationship between estimated and } \\
\text { observed RSS [75] }\end{array}$ & $\begin{array}{l}\text { Construction of the Feature-Vector } \\
\text { Representation [76], AP clustering [77] }\end{array}$ \\
\hline $\begin{array}{l}\text { Coverage } \\
\text { Area }\end{array}$ & $\begin{array}{l}\text { There are three levels of coverage: local, } \\
\text { scalable, and global, existing INSs } \\
\text { ranges are usually from } 5 \text { to } 50 \mathrm{~m} \text {, } \\
\text { coverage is closely related to accuracy }\end{array}$ & $\begin{array}{l}\text { Wi-Fi fingerprint localization [78], } \\
\text { horizontal placement i Beacon [79] }\end{array}$ & $\begin{array}{l}\text { RP Clustering and Coarse Localization } \\
\text { [80-82]Learning the possibility of } \\
\text { moving each node to reduce the number } \\
\text { of individual nodes as much as possible } \\
\text { based on virtual areas by selecting } \\
\text { nodes based on learning among nodes. } \\
\text { [83] }\end{array}$ \\
\hline Scalability & $\begin{array}{l}\text { Scalability of an INS means the system } \\
\text { ensures the normal positioning function } \\
\text { when it scales in one of two } \\
\text { dimensions: geography and number of } \\
\text { users, indicates that the number of units } \\
\text { located per time period per geographic } \\
\text { area increases }\end{array}$ & $\begin{array}{l}\text { Location-Aware Communications for } 5 \mathrm{G} \\
\text { Networks [84], BLE profile adaptor } \\
\text { uses DDS [85] }\end{array}$ & $\begin{array}{l}\text { Multi-Agent paradigm-the high number } \\
\text { of sensors which requires flexible } \\
\text { communication mechanisms to } \\
\text { guarantee scalability [86], } \\
\text { Infrastructure Perspective [87] }\end{array}$ \\
\hline Cost & Money, time, space, and energy & $\begin{array}{l}\text { This technique can be implemented with } \\
\text { WiFi, BLE and Zig bee, i Beacon } \\
\text { standard introduced by Apple is based } \\
\text { on RSSI [61] }\end{array}$ & $\begin{array}{l}\text { Cost estimation algorithm and decision- } \\
\text { making model [88] }\end{array}$ \\
\hline Privacy & Protect data from intrusion & $\begin{array}{l}\text { Location-privacy preserving mechanism } \\
\text { (LPPM) [89] }\end{array}$ & $\begin{array}{l}\text { Collect and analyze sensitive data that is } \\
\text { governed by privacy rules [90], The } \\
\text { joint use of end-to-end security and } \\
\text { identication of anomalous } \\
\text { measurements to provide security for } \\
\text { IoT devices directly connected to the } \\
\text { Internet [91] }\end{array}$ \\
\hline
\end{tabular}

accuracy [67]. Tom Hought and colleagues evaluated the performance of 24 IPSs in a modern health care environment. Evaluation (data collection and data processing) has been executed using standard techniques with spot accuracy, room position accuracy, and multiple IPS delays. to investigate the solutions, the location of a fixed device in 73 locations was examined and using the same evaluation locations for all IPS, The performance of all systems was compared and, consequently, several trends were substantially cheaper to run, including a WiFi solution based on the pattern of effect that has the optimum result (accuracy point 1.21 meters and room accuracy 98\%) and proximity based on the solution (based on sensor nodes) [68].

A wireless positioning approach was presented by Wei Zhang and co-workers which is based on deep learning. to manage the numerous and unforeseen kinds of wireless signals in the four layers of the deep neural network, the pre-trained structure of automated noise removal can learn the trusted qualities of a large set of noise-free samples and also to preserve uniformity. The hidden model of Markov has been presented. The main issue in pattern matching is the internal situation of the diversity of RSSIs caused by the unstable nature of wireless signals, which include 
multi-path damping and fading by moving and fixed objects [92]. Furthermore, Voice sensors on a mobile phone can identify any disruption in a persons voice in comparison to a series of published sounds and thus identify a number of health problems in a person. Eventually, all cyber-like features of people, like physical, social and psychological intelligences are interacting in the smart world [93]. In Machine Learning method, there is a data set which is trained employing a model and finally the model trained to foresee new data [92].In order to handle the various and unpredictable types of wireless signals in the four layers of the deep Neural Network (DNN), the pertrained structure is introduced by the Automated Noise Reduction (SDA) ability, which can learn the reliable characteristics of a large set of noise-proof and also to maintain the integrity of the Hidden Markof Model (HMM). Crowd Sensing for INS is a paradigm for reaching advanced method making use of population shares, which is highly appropriate for Finger Print based on INS. In [94], Position algorithms, state-of-the-art solutions and challenges with regards to basic knowledge are mentioned. To attain a PDR, method and signal amplification with the SLAM algorithm were merged in order to acquire the signal map and user path at the same time. Clearly data integration is the most demanding job with growing population. Data quality control algorithms and merger algorithms are at the moment being ignored. Researchers have become more accurate towards the combination of large data and signal attributes.

Table 7 summarizes the primary research problems with INS for which DM solutions have been utilized. In order to solve vehicle navigation problems in [95] made use of Fuzzy logic which is typically utilized for classification with decision tree, they offered FDT algorithm for pedestrian. It can offer satisfactory accuracy and has seamless navigation with lower complexity. Yaqin Xie et al feed the RSSI values as fingerprints in order to shape the radio map used in the training process, they merge spear-man distance base on the rank correlation coefficient with the original KNN approach, outcome indicate that the suggested combination technique leads to greater performance.

In [96] using clustering was introduced for problem of coordinated robot navigation. According to hierarchical clustering, they offered a natural abstraction for ensemble task encoding and control with regards to accurate yet flexible organizational features at different resolutions. In many papers a realistic indoor multi path environment classification developed for instance the approach used in [97] was according to statistics of correlation which were related to system bandwidth and grid spacing that illustrates a certain pattern that differentiates LOS open space and LOS cluttered. The outcomes are essential for creating a framework for developing a database of RF channel fingerprints for indoor multi path environment classification. In [98] With the purpose of considering the complexity of indoor environment ensemble algorithm based on regression tree in order to improve the accuracy, the approach provided low localization error and the suggested strategy seems that the computation cost is decreased by utilizing smaller training subsets. Zhefu Wu et al designed a Naive Bayes classifier-based indoor localization system with propose to use CSI, their technique choose the most reliable ones for the localization with reach $90 \%$ classification accuracy. [99] Offered a kind of indoor matching algorithm based on KNN and a multi-later constraint which the accuracy of navigation system is improved by more than $55 \%$ with only $0.4 \%$ final position error. There are a number of other research in this field which demonstrates using data mining methods to solve the INS problems.

\section{Concluding remarks \& future work}

Accuracy/accuracy, availability, coverage, and minimal costs for local installations are the requirements for application environments. In order to get over this disadvantage, a great portion of research approaches is needed to deal with such problems. Considering the fast growth of IoT, the biggest challenge is to examine the huge volumes of data and draw out valuable knowledge or information for future measures [109]. Data investments are still rising but are also exhibiting some indications of decline.

The idea is transitioning toward certain effects to business metrics and areas. This process of normalization can reintroduce IT problems regarding leadership and skills for data and leaders involved in analytics. There are many problems when IoT, data mining and Indoor Navigation System get together:

Accuracy Accuracy continues to be a highly difficult area for numerous researchers. Although the main driver for the majority of applications is INS accuracy, some compromises may need to be made between accuracy and some other performance metrics. Then we focused on the studies which employ DM-based approaches for the indoor navigation systems

Complexity The knowledge necessary to extract: the knowledge is significantly covered in big amounts of data the knowledge is also not simple. Thus we have to examine the attributes of data finding the organization of them.

Data sharing How to mine incomplete and unclear data with the aim of data applications. In data mining system, one of the biggest difficulties is choosing an elective and security solution to share data between various applications and systems, due to the fact sensitive information, like medical records and banking transactions, must be highly considered. 
Table 7 The main research problems in INS that DM solutions have been used

\begin{tabular}{|c|c|c|}
\hline $\begin{array}{l}\text { DM } \\
\text { Techniques }\end{array}$ & Main target problems in INS & REF \\
\hline Decision tree & $\begin{array}{l}\text { Reduce the accumulated error, improve the generation ability and minimize the use of infrastructure with fuzzy } \\
\text { decision tree (FDT) }\end{array}$ & [95] \\
\hline $\mathrm{KNN}$ & $\begin{array}{l}\text { Improve accuracy, adaptation and provide continuous position information based on k-nearest neighbor algorithm } \\
\text { (KNN) }\end{array}$ & [100] \\
\hline \multirow[t]{2}{*}{ SVM } & $\begin{array}{l}\text { Increase the accuracy and decrease the costs, robust against noisy and large data set with a hybrid of the k-nearest } \\
\text { neighbors algorithm and the Multi-Class Support Vector Machines (KNN-SVM) model }\end{array}$ & [99] \\
\hline & Coarse-grained turn estimation can be performed with very high accuracy & [101] \\
\hline Clustering & $\begin{array}{l}\text { Offers a formalism for identifying with use of hierarchical effective reactive algorithms for navigating through the } \\
\text { combinatorial space in concert with geometric realizations for a particular choice of the hierarchical clustering } \\
\text { method }\end{array}$ & [96] \\
\hline k-means & $\begin{array}{l}\text { K-Means clustering is proposed to automatically identify and discard transient high amplitude interferences and make } \\
\text { noise covariances estimation }\end{array}$ & [102] \\
\hline Classification & $\begin{array}{l}\text { A realistic indoor multi path environment classification based on practical RF measurements that is a compromise } \\
\text { between accuracy and resources/complexity }\end{array}$ & [97] \\
\hline \multirow[t]{2}{*}{ Regression } & $\begin{array}{l}\text { Improve robustness and accuracy, localization error and the computation complexity based on regression tree } \\
\text { Improvement in the positional accuracy base on Artificial Neural Network (ANN) }\end{array}$ & [98] \\
\hline & Support Vector Machine Regression (SVR) and Partial Least Squares Regression (PLSR) & [103] \\
\hline $\begin{array}{l}\text { Bayesian } \\
\text { networks }\end{array}$ & $\begin{array}{l}\text { Accurate localization and efficient navigation for emergency evacuation in indoor spaces based on Bayesian inference } \\
\text { localization }\end{array}$ & [104] \\
\hline \multirow[t]{2}{*}{ Nave Bayes } & $\begin{array}{l}\text { Naive Bayes technique for localization provides better accuracy, resolves zero probability issue caused due to data } \\
\text { incompleteness }\end{array}$ & [105] \\
\hline & Develop a Naive Bayes classifier to Improve localization accuracy(environment noises, multi path effect) & [106] \\
\hline \multirow[t]{2}{*}{ Deep learning } & Complex data handling processes base on deep learning approach evaluation & [107] \\
\hline & $\begin{array}{l}\text { Understanding the correlation and causality between actions of an agent and the changes of the environment as a result } \\
\text { of the action }\end{array}$ & [108] \\
\hline
\end{tabular}

Access The challenge here is to access, taking out large scale data from various locations of data storage. Here, we have to deal with the heterogeneity, variety, as well as noise of the data. Moreover, it is a major problem to find the errors and even more challenging to remove them. How to alter traditional algorithms to large data environment is a major difficulty in data mining algorithms area.

Security Data mining system, privacy is very critical. Privacy and security safeguard the data from illegal access and privacy intrusion. Data mining system Security makes data integrating, sharing, and mining more available to the third part of developer.

This study regards strong and accurate indoor localization as a major enabler of services which are locationbased. Real-time interaction between objects, users, and Indoor Navigation systems, which includes the need for accurate identification of activities and objects, is the major function this kind of applications require. The paper deals with remarkably accurate and efficient indoor positioning as faced in indoor environments. Robust and accurate indoor positioning is an important enabler for a wealth of future location-based services, which range from supplychain administration and producing to health-care and leisure activities. Accuracy will empower a numerous modern location-based applications due to the fact such accuracy levels basically allow for a practical interaction in real-time between humans and indoor physical systems. Data Mining enabling technologies like advanced multiprocessor computers, algorithms, huge databases with attributes like proactive, prospective information delivery.

\section{Conclusion}

This study presented a comprehensive literature review on data mining techniques for Indoor Navigation Systems in IoT based scenarios. This ability paves the way for influencing many applications areas, ranging from navigation to marketing. Use of data mining techniques for any collection of data sets is so big and complicated that it will become hard to process using traditional data processing applications to the data analytics: the ways we draw out information from the data, calculate it, and offer it for decision-making. Data mining technologies are merged with IoT technologies related to decision making. With data mining techniques, indoor navigation systems are able 
to make more efficient decisions regarding marketing, advertising, etc. these decisions will enable IPS to expand themselves. This study is valuable for indoor localization systems, for which a reduction of the necessary infrastructure is very significant while maintaining the needed level of accuracy and strength. Data Mining can be employed in various sectors of Indoor Positioning system in order to both predict and discover trends. It is a proactive solution for IPS looking to obtain a competitive advantage.

\section{References}

1. Thibaud, M., Chi, H., Zhou, W., \& Piramuthu, S. (2018). Internet of things (iot) in high-risk environment, health and safety (ehs) industries: A comprehensive review, Decision Support Systems.

2. Kumar, P. M., Gandhi, U., Varatharajan, R., Manogaran, G., Jidhesh, R., \& Vadivel, T. (2017). Intelligent face recognition and navigation system using neural learning for smart security in internet of things, Cluster Computing, pp. 1-12.

3. Shu, Y., Shin, K. G., He, T., \& Chen, J. (2015). Last-mile navigation using smartphones, In Proceedings of the 21 st annual international conference on mobile computing and networking, ser. MobiCom '15. New York, NY, USA: ACM, 2015, pp. 512-524. [Online]. Available: https://doi.org/10.1145/ 2789168.2790099

4. Xiao, Z., Wen, H., Markham, A., \& Trigoni, N. (2014). Lightweight map matching for indoor localisation using conditional random fields, In Proceedings of the 13th international symposium on information processing in sensor networks, ser. IPSN '14. Piscataway, NJ, USA: IEEE Press, 2014, pp. 131-142. [Online]. Available: http://dl.acm.org/citation.cfm?id=2602339. 2602355

5. Farahzadi, A., Shams, P., Rezazadeh, J., \& Farahbakhsh, R. (2017). Middleware technologies for cloud of things-a survey.Digital Communications and Networks, Elsevier.

6. Wlodarczak, P., Ally, M., \& Soar, J. (2017). Data mining in iot: Data analysis for a new paradigm on the internet, In Proceedings of the international conference on web intelligence (pp. 1100-1103). ACM, 2017.

7. He, S., Chan, S. H. G., Yu, L., \& Liu, N. (April 2015). Fusing noisy fingerprints with distance bounds for indoor localization, In 2015 IEEE conference on computer communications (INFOCOM) (pp. 2506-2514) April 2015.

8. REzazadeh, J., Moradi, M., Ismail, A. S., \& Dutkiewicz, E. (2014). Superior path planning mechanism for mobile beaconassisted localization in wireless sensor networks. IEEE Sensors Journal, 14, 3052-3064.

9. Fleury, A., Vacher, M., \& Noury, N. (2010). Svm-based multimodal classification of activities of daily living in health smart homes: Sensors, algorithms, and first experimental result s. IEEE Transactions on Information Technology in Biomedicine, 14(2), 274-283.

10. Win, M. Z., Conti, A., Mazuelas, S., Shen, Y., Gifford, W. M., Dardari, D., \& Chiani, M. (2011). Network localization and navigation via cooperation. IEEE Communications Magazine, vol. 49 , no. 5 .

11. Fayyad, U., Piatetsky-Shapiro, G., \& Smyth, P. (1996). The kdd process for extracting useful knowledge from volumes of data. Communications of the ACM, 39(11), 27-34. https://doi.org/10. $1145 / 240455.240464$
12. Han, J., Pei, J., \& Kamber, M. (2011). Data mining: Concepts and techniques. New York: Elsevier.

13. Shearer, C. (2000). The crisp-dm model: The new blueprint for data mining. Journal of Data Warehousing, 5(4), 13-22.

14. Kumar, S., Qadeer, M. A., \& Gupta, A. (2009). Location based services using android (lbsoid). In 2009 IEEE international conference on internet multimedia services architecture and applications (IMSAA) (pp. 1-5) IEEE, 2009.

15. Summers, I. R., \& Chanter, C. M. (2002). A broadband tactile array on the fingertip. The Journal of the Acoustical Society of America, 112(5), 2118-2126.

16. Beder, C., \& Klepal, M. (2012). Fingerprinting based localisation revisited: A rigorous approach for comparing rssi measurements coping with missed access points and differing antenna attenuations. In 2012 international conference on indoor positioning and indoor navigation (IPIN) (pp. 1-7) IEEE, 2012.

17. Kay, S. M. (1993). Fundamentals of statistical signal processing. Englewood Cliffs: Prentice-Hall.

18. Shen, Y., Mazuelas, S., \& Win, M. Z. (2012). Network navigation: Theory and interpretation. IEEE Journal on Selected Areas in Communications, 30(9), 1823-1834.

19. Paull, L., Saeedi, S., Seto, M., \& Li, H. (2014). Auv navigation and localization: A review. IEEE Journal of Oceanic Engineering, 39(1), 131-149.

20. Zheng, Y., Shen, G., Li, L., Zhao, C., Li, M., \& Zhao, F. (2017). Travi-navi: Self-deployable indoor navigation system. IEEE/ ACM Transactions on Networking, 25(5), 2655-2669.

21. Rezazadeh, J., Moradi, M., \& Ismail, A. S. (2012). Messageefficient localization in mobile wireless sensor networks. Jour nal of Communication and Computer (JCC), 9(3), 340-344.

22. Yang, Z., Wu, C., Zhou, Z., Zhang, X., Wang, X., \& Liu, Y. (2015). Mobility increases localizability: A survey on wireless indoor localization using inertial sensors. ACM Computing Surveys (CSUR), 47(3), 54.

23. Sanchez, J., \& Saenz, M. (2008). Orientation and mobility in external spaces for blind apprentices using mobile devices. Mag Ann Metrop Univ, 8, 47-66.

24. Hossain, A. M., \& Soh, W.-S. (2015). A survey of calibrationfree indoor positioning systems. Computer Communications, 66, $1-13$.

25. Sonnenblick, Y. (1998). An indoor navigation system for blind individuals, In Proceedings of the 13th annual conference on technology and persons with disabilities (pp. 215-224).

26. Faragher, R., \& Harle, R. (2015). Location fingerprinting with bluetooth low energy beacons. IEEE Journal on Selected Areas in Communications, 33(11), 2418-2428.

27. He, S., Lin, W., \& Chan, S.-H. (2016). Indoor localization and automatic fingerprint update with altered ap signals. IEEE Transactions on Mobile Computing, 16, 1897-1910.

28. Halder, S., \& Ghosal, A. (2016). A survey on mobile anchor assisted localization techniques in wireless sensor networks. Wireless Networks, 22(7), 2317-2336.

29. Kang, J.-H., Kwon, Y.-M., \& Park, K.-J. (2017). Cooperative spatial retreat for resilient drone networks. Sensors, 17(5), 1018.

30. Sukkarieh, S., Nebot, E. M., \& Durrant-Whyte, H. F. (2012). The gps aiding of ins. In Field and service robotics. Springer, 2012, 267.

31. Dardari, D., Closas, P., \& Djurić, P. M. (2015). Indoor tracking: Theory, methods, and technologies. IEEE Transactions on Vehicular Technology, 64(4), 1263-1278.

32. Kessel, M., \& Werner, M. (2011). Smartpos: Accurate and precise indoor positioning on mobile phones. In Proceedings of the first international conference on mobile services, resources, and users, MOBILITY 2011, pp. 158-163. 
33. Liu, H., Darabi, H., Banerjee, P., \& Liu, J. (2007). Survey of wireless indoor positioning techniques and systems. IEEE Transactions on Systems, Man, and Cybernetics Part C (Applications and Reviews), 37(6), 1067-1080.

34. Durrant-Whyte, H. (2001). A critical review of the state-of-theart in autonomous land vehicle systems and technology. Albuquerque (NM) and Livermore (CA), USA: SandiaNationalLaboratories, vol. 41.

35. Rezazadeh, J., Moradi, M., \& Ismail, A. S. (2012). Mobile wireless sensor networks overview. International Journal of Computer Communications and Networks, 2(1), 17-22.

36. Rezazadeh, J., Moradi, M., Ismail, A. S., \& Dutkiewicz, E. (2015). Impact of static trajectories on localization in wireless sensor networks. Wireless Networks, 21(3), 809-827.

37. Rezazadeh, J., Moradi, M., \& Ismail, A. S. (2012). Fundamental metrics for wireless sensor networks localization. International Journal of Electrical and Computer Engineering (IJECE), 2(4), 452-455.

38. Wang, J., Ghosh, R. K., \& Das, S. K. (2010). A survey on sensor localization. Journal of Control Theory and Applications, 8(1), $2-11$.

39. Alrajeh, N. A., Bashir, M., \& Shams, B. (2013). Localization techniques in wireless sensor networks. International Journal of Distributed Sensor Networks, 2013, 1-9.

40. Rezazadeh, J., Moradi, M., \& Ismail, A. (2011). Efficient localization via middle-node cooperation in wireless sensor networks. In 2011 international conference on electrical, control and computer engineering (INECCE), 2011, pp. 410-415.

41. Moradi, M., Rezazadeh, J., \& Ismail, A. S. (2012). A reverse localization scheme for underwater acoustic sensor networks. Sensors, 12, 4352-4380.

42. Chen, Z., Zhu, Q., \& Soh, Y. C. (2016). Smartphone inertial sensor-based indoor localization and tracking with ibeacon corrections. IEEE Transactions on Industrial Informatics, 12(4), $1540-1549$.

43. Nguyen, K. A. \& Luo, Z. (2017). On assessing the positioning accuracy of google tango in challenging indoor environments. In 2017 international conference on indoor positioning and indoor navigation (IPIN) (pp. 1-8). IEEE, 2017.

44. Neges, M., Koch, C., König, M., \& Abramovici, M. (2017). Combining visual natural markers and imu for improved ar based indoor navigation. Advanced Engineering Informatics, 31, $18-31$.

45. Ryu, J. H., Gankhuyag, G., \& Chong, K. T. (2016). Navigation system heading and position accuracy improvement through gps and ins data fusion. Journal of Sensors, 9, 1-9.

46. Hu, P., Wang, S., Zhang, R., Liu, X., \& Xu, B. (2017). Fast heading-rotation-based high-accuracy misalignment angle estimation method for ins and gnss. Measurement, 102, 208-213.

47. Yue, Z., Lian, B., \& Tang, C. (2017). The gps/ins integrated navigation method based on adaptive ssr-sckf cubature kalman filter. In China satellite navigation conference (pp. 395-405). Springer, 2017.

48. Zhao, J., Pei, F. -j., \& Liu, H. -y., (2016). Self-alignment for strapdown ins using real-time adaptive filter. In 2016 IEEE Chinese guidance, navigation and control conference (CGNCC) (pp. 2022-2026). IEEE, 2016.

49. Fayyad, U., Piatetsky-Shapiro, G., \& Smyth, P. (1996). The kdd process for extracting useful knowledge from volumes of data. Communications of the ACM, 39(11), 27-34.

50. Hall, M., Frank, E., Holmes, G., Pfahringer, B., Reutemann, P., \& Witten, I. H. (2009). The weka data mining software: An update. ACM SIGKDD Explorations Newsletter, 11(1), 10-18.

51. Team, R. C. (2000). R language definition. Vienna, Austria: R foundation for statistical computing.
52. Graczyk, M., Lasota, T., \& Trawiński, B. (2009). Comparative analysis of premises valuation models using keel, rapidminer, and weka. In International conference on computational collective intelligence (pp. 800-812). Springer, 2009.

53. Yu, Q., \& Shen, Y. (2016). Research of information security risk prediction based on grey theory and anp. In Advanced information management, communicates, electronic and automation control conference (IMCEC) (pp. 107-113). IEEE, 2016.

54. Luo, J., \& Bridges, S. M. (2000). Mining fuzzy association rules and fuzzy frequency episodes for intrusion detection. International Journal of Intelligent Systems, 15(8), 687-703.

55. Guyot, A., Hubert-Moy, L., \& Lorho, T. (2018). Detecting neolithic burial mounds from lidar-derived elevation data using a multi-scale approach and machine learning techniques. Remote Sensing, 10(2), 225.

56. Llamas, J., Lerones, P. M., Medina, R., Zalama, E., \& GómezGarcía-Bermejo, J. (2017). Classification of architectural heritage images using deep learning techniques. Applied Sciences, 7(10), 992.

57. Wang, X., Gao, L., Mao, S., \& Pandey, S. (2017). Csi-based fingerprinting for indoor localization: A deep learning approach. IEEE Transactions on Vehicular Technology, 66(1), 763-776.

58. Kumar, A. K. T. R., Schäufele, B., Becker, D., Sawade, O., \& Radusch, I. (2016). Indoor localization of vehicles using deep learning, In 2016 IEEE 17th international symposium on a world of wireless, mobile and multimedia networks (WoWMoM) (pp. 1-6). IEEE, 2016.

59. Litjens, G., Kooi, T., Bejnordi, B. E., Setio, A. A. A., Ciompi, F., Ghafoorian, M., et al. (2017). A survey on deep learning in medical image analysis. Medical Image Analysis, 42, 60-88.

60. Siddiqa, A., Hashem, I. A. T., Yaqoob, I., Marjani, M., Shamshirband, S., Gani, A., et al. (2016). A survey of big data management: Taxonomy and state-of-the-art. Journal of Network and Computer Applications, 71, 151-166.

61. Gui, L., Yang, M., Fang, P., \& Yang, S. (2017). Rss-based indoor localization using multiplicative distance-correction factor. IET Wireless Sensor Systems, 7, 98-104.

62. Yassin, A., Nasser, Y., Awad, M., Al-Dubai, A., Liu, R., Yuen, C., et al. (2016). Recent advances in indoor localization: A survey on theoretical approaches and applications. IEEE Communications Surveys \& Tutorials., 19, 1327-1346.

63. Zhu, C., Leung, V. C., Shu, L., \& Ngai, E. C.-H. (2015). Green internet of things for smart world. IEEE Access, 3, 2151-2162.

64. Zafari, F., Papapanagiotou, I., Devetsikiotis, M., \& Hacker, T. J. (2017). Enhancing the accuracy of ibeacons for indoor proximity-based services. In IEEE ICC.

65. Chen, L. -K., \& Hong, Y. (2016). A vision-based indoor positioning method with high accuracy and efficiency based on selfoptimized-ordered visual vocabulary. In 2016 IEEE/ION position, location and navigation symposium (PLANS) (pp. 48-56). IEEE, 2016.

66. Wang, H., Zhao, Z., Hu, J., Qu, Z., \& Feng, H. (2016). Study on improvement of fingerprint matching algorithm in wireless lan based indoor positioning system. In 2016 17th IEEE/ACIS international conference on software engineering, artificial intelligence, networking and parallel/distributed computing (SNPD) (pp. 275-280). IEEE, 2016.

67. Yun, S., Yao, Z., Wang, T., \& Lu, M. (2016). High accuracy and fast acquisition algorithm for pseudolites-based indoor positioning systems. In 2016 fourth international conference on ubiquitous positioning, indoor navigation and location based services (UPINLBS) (pp. 51-60). IEEE, 2016.

68. Pérez, S., Laperrière, V., Borderon, M., Padilla, C., Maignant, G., \& Oliveau, S. (2016). Evolution of research in health geographics through the international journal of health geographics 
(2002-2015). International Journal of Health Geographics, 15(1), 3.

69. Khalajmehrabadi, A., Gatsis, N., \& Akopian, D. (2016). Modern wlan fingerprinting indoor positioning methods and deployment challenges. arXiv preprint arXiv:1610.05424.

70. George, J., Kumar, V., \& Kumar, S. (2015). Data warehouse design considerations for a healthcare business intelligence system. In World congress on engineering

71. Lee, C. K., Ho, W., Ho, G. T., \& Lau, H. C. (2011). Design and development of logistics workflow systems for demand management with rfid. Expert Systems With Applications, 38(5), 5428-5437.

72. Peng, Y., Kou, G., Wang, G., Wu, W., \& Shi, Y. (2011). Ensemble of software defect predictors: An ahp-based evaluation method. International Journal of Information Technology \& Decision Making, 10(01), 187-206.

73. García-Osorio, C., de Haro-García, A., \& García-Pedrajas, N. (2010). Democratic instance selection: A linear complexity instance selection algorithm based on classifier ensemble concepts. Artificial Intelligence, 174(5), 410-441.

74. Mishra, D. P., Samantaray, S. R., \& Joos, G. (2016). A combined wavelet and data-mining based intelligent protection scheme for microgrid. IEEE Transactions on Smart Grid, 7(5), 2295-2304.

75. Gu, Y., Song, Q., Ma, M., Li, Y., \& Zhou, Z. (2016). Using ibeacons for trajectory initialization and calibration in footmounted inertial pedestrian positioning systems. In 2016 International Conference on Indoor Positioning and Indoor Navigation (IPIN) (pp. 1-7). IEEE, 2016.

76. Kazienko, P., Musial, K., \& Kajdanowicz, T. (2011). Multidimensional social network in the social recommender system. IEEE Transactions on Systems, Man, and Cybernetics-Part A: Systems and Humans, 41(4), 746-759.

77. Rodriguez, A., \& Laio, A. (2014). Clustering by fast search and find of density peaks. Science, 344(6191), 1492-1496.

78. He, S., \& Chan, S.-H. G. (2016). Wi-fi fingerprint-based indoor positioning: Recent advances and comparisons. IEEE Communications Surveys \& Tutorials, 18(1), 466-490.

79. Vossen, G., Dillon, S., Schomm, F., \& Stahl, F. (2017). A classification framework for beacon applications. Open Journal of Internet of Things (OJIOT), 3(1), 1-11.

80. Kushki, A., Plataniotis, K. N., \& Venetsanopoulos, A. N. (2010). Intelligent dynamic radio tracking in indoor wireless local area networks. IEEE Transactions on Mobile Computing, 9(3), 405-419.

81. Han, D., Jung, S., Lee, M., \& Yoon, G. (2014). Building a practical wi-fi-based indoor navigation system. IEEE Pervasive Computing, 13(2), 72-79.

82. Awang, A., \& Agarwal, S. (2015). Data aggregation using dynamic selection of aggregation points based on rssi for wireless sensor networks. Wireless Personal Communications, 80(2), 611-633.

83. Park, J., Ryu, J., \& Yang, S. -B. Activedbc: Learning knowledge-based information propagation in mobile social networks. Wireless Networks pp. 1-13.

84. Di Taranto, R., Muppirisetty, S., Raulefs, R., Slock, D., Svensson, T., \& Wymeersch, H. (2014). Location-aware communications for $5 \mathrm{~g}$ networks: How location information can improve scalability, latency, and robustness of 5g. IEEE Signal Processing Magazine, 31(6), 102-112.

85. Oh, J. -H., Back, M. -K., Oh, G. -T., \& Lee, K. -C. (2016). A study on dds-based ble profile adaptor for solving ble data heterogeneity in internet of things. In International conference on computer science and its applications (pp. 619-624) Springer, 2016.
86. Shi, D., Zurada, J., \& Guan, J. (2014). A neuro-fuzzy approach to bad debt recovery in healthcare, In System Sciences (HICSS), 2014 47th Hawaii International Conference on IEEE, 2014, pp. 2888-2897.

87. Tsai, C.-W., Lai, C.-F., Chiang, M.-C., Yang, L. T., et al. (2014). Data mining for internet of things: A survey. IEEE Communications Surveys and Tutorials, 16(1), 77-97.

88. Różewski, P., \& Małachowski, B. (2009). Competence management in knowledge-based organisation: case study based on higher education organisation, In International conference on knowledge science, engineering and management (pp. 358-369). Springer, 2009 .

89. Shokri, R., Theodorakopoulos, G., Troncoso, C., Hubaux, J. -P. \& Le Boudec, J. -Y. (2012). Protecting location privacy: Optimal strategy against localization attacks. In Proceedings of the 2012 ACM conference on computer and communications security (pp. 617-627). ACM, 2012.

90. Cucoranu, I. C., Parwani, A. V., West, A. J., Romero-Lauro, G., Nauman, K., Carter, A. B., et al. (2013). Privacy and security of patient data in the pathology laboratory. Journal of Pathology Informatics, 4(1), 4.

91. Junior, N. F., Silva, A., Guelfi, A., \& Kofuji, S. T. (2017). Iot6sec: Reliability model for internet of things security focused on anomalous measurements identification with energy analysis. Wireless Networks pp. 1-24.

92. Zhang, W., Liu, K., Zhang, W., Zhang, Y., \& Gu, J. (2016). Deep neural networks for wireless localization in indoor and outdoor environments. Neurocomputing, 194, 279-287.

93. Kopetz, H. (2011). Internet of things. In Real-time systems (pp. 307-323). Springer, 2011.

94. Pei, L., Zhang, M., Zou, D., Chen, R., \& Chen, Y. (2016). A survey of crowd sensing opportunistic signals for indoor localization. Mobile Information Systems, 2016, 1-16.

95. Liao, J. -K., Chiang, K. -W., Tsai, G. -J., \& Chang, H. -W. (2016). A low complexity map-aided fuzzy decision tree for pedestrian indoor/outdoor navigation using smartphone. In 2016 international conference on indoor positioning and indoor navigation (IPIN) (pp. 1-8). IEEE, 2016.

96. Arslan, O., Guralnik, D. P., \& Koditschek, D. E. (2016). Coordinated robot navigation via hierarchical clustering. IEEE Transactions on Robotics, 32(2), 352-371.

97. Alhajri, M., Alsindi, N., Ali, N., \& Shubair, R. (2016). Classification of indoor environments based on spatial correlation of $\mathrm{rf}$ channel fingerprints. In 2016 IEEE international symposium on antennas and propagation (APSURSI) (pp. 1447-1448). IEEE, 2016.

98. Ahmadi, H., Viani, F., Polo, A., \& Bouallegue, R. (2016). An accurate ensemble-based wireless localization strategy for wireless sensor networks, In 2016 24th international conference on software, telecommunications and computer networks (SoftCOM) (pp. 1-5). IEEE, 2016.

99. Xin, H., Zhi, X., Jianxin, X., \& Limin, X. (2017). Research on pedestrian navigation system aided by indoor geomagnetic matching. In 2017 29th Chinese control and decision conference (CCDC) (pp. 1946-1951). IEEE, 2017.

100. Tabrizi, S. S., \& Cavus, N. (2016). A hybrid knn-svm model for iranian license plate recognition. Procedia Computer Science, 102, 588-594.

101. Gonzalez, E. J., Luo, C., Shrivastava, A., Palem, K., Moon, Y., Noh, S., et al. (2017). Location detection for navigation using imus with a map through coarse-grained machine learning, In 2017 design, automation \& test in Europe conference \& exhibition (DATE) (pp. 500-505). IEEE, 2017.

102. Chen, R. and (2017). A study of gps/ins integrated navigation with artificial neural network and k-means aided kalman filter. HKU Theses Online (HKUTO). 
103. Xiao, Z., Zhan, S., Xiang, Z., Wang, D., \& Chen, W. (2016). A gpr-pso incremental regression framework on gps/ins integration for vehicle localization under urban environment. In 2016 IEEE 27th annual international symposium on personal, indoor, and mobile radio communications (PIMRC) (pp. 1-6). IEEE, 2016.

104. Wang, W., \& Ku, W.-S. (2017). Dynamic indoor navigation with bayesian filters. SIGSPATIAL Special, 8(3), 9-10.

105. Haq, M. A. U., Kamboh, H. M. A., Akram, U., Sohail, A., \& Iram, H. (2016). Indoor localization using improved multinomial naïve bayes technique. In International afro-European conference for industrial advancement (pp. 321-329) .Springer, 2016.

106. Wu, Z., Xu, Q., Li, J., Fu, C., Xuan, Q., \& Xiang, Y. (2017). Passive indoor localization based on csi and naive bayes classification. IEEE Transactions on Systems, Man, and Cybernetics: Systems, 49, 1-12.

107. Perera, L. P., \& Mo, B. (2016). Data compression of ship performance and navigation information under deep learning. In Proceedings of the 35th international conference on ocean, offshore and arctic engineering (OMAE 2016), Busan, Korea, (OMAE2016-54093).

108. Zhu, Y., Mottaghi, R., Kolve, E., Lim, J. J., Gupta, A., Fei-Fei, L., \& Farhadi, A. (2017). Target-driven visual navigation in indoor scenes using deep reinforcement learning. In 2017 IEEE international conference on robotics and automation (ICRA)(pp. 3357-3364). IEEE, 2017.

109. Pratiba, D., \& Shobha, G. (2014). A survey of resource sharing cloud using data mining, In 2014 fifth international conference on signal and image processing (ICSIP) (pp. 323-327). IEEE, 2014.

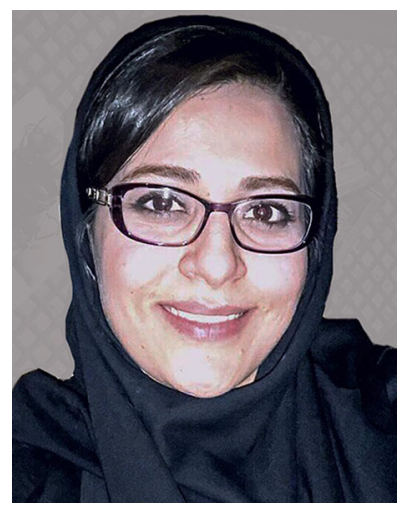

Mahbubeh Sattarian received the M.S. degree in Information Technology with specialization in Computer Networks from Islamic Azad University, Tehran North Branch, Iran. Her research interests include Indoor Navigation System for health monitoring, indoor localization, data mining and machine learning.

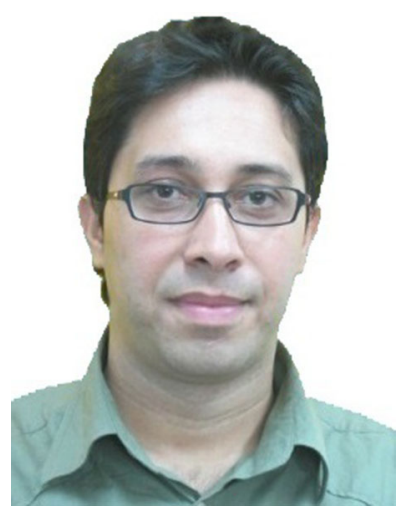

Javad Rezazadeh is currently a researcher at University of Technology, Sydney (UTS) in Australia and also an assistant professor at the Islamic Azad University of North Tehran Branch in Iran. He holds a Ph.D. in Wireless Communications from University Technology Malaysia (UTM) in 2014. He received his M.S. and B.S. Degrees in Software Engineering in 2008 and 2005 respectively. He was a recipient of the Academic Excellent Student Award for the top 1 postgraduate in the year 2014. His research interests include localization Systems, Internet of Things (IoT), Smart Cities and Wireless Networks and Mobile Computing.

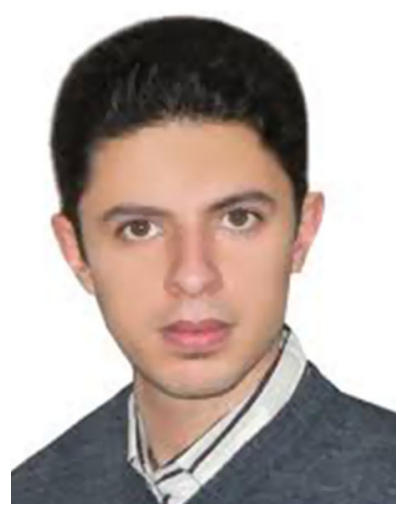

Behavior Analysis.

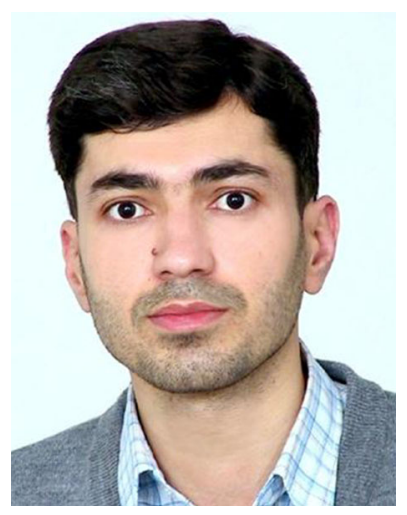

Reza Farahbakhsh is currently a research fellow at Institut-Mines Telecom, Telecom SudParis. He received his $\mathrm{Ph} . \mathrm{D}$. degree from Institut-Mines Telecom, Telecom SudParis (CNRS Lab UMR5157) jointly with Paris VI (UPMC) at 2015, his M.S. in Computer Engineering degree from University of Isfahan, Iran, 2008 and B.S. degree from Qazvin Azad University in 2006. His research interest is Online Social networks, IoT, Large scale measurement, User

Alireza Bagheri is currently an assistant professor in the computer engineering and IT Department at Amirkabir University of Technology (AUT) at Tehran. He received his Ph.D. degree in computer science from Amirkabir University of Technology (AUT) at Tehran, in 2005, his M.S. and B.S. degrees in computer engineering from Sharif University of Technology (SUT) at Tehran, respectively in 1998 and 1996. His research interests include computational geometry, graph drawing and graph algorithms. 